\title{
MULTIWAVELENGTH OBSERVATIONS OF THE LOW-METALLICITY BLUE COMPACT DWARF GALAXY SBS $0335-052^{1}$
}

\author{
Daniel A. Dale, ${ }^{2}$ George Helou,,${ }^{3,4}$ Gerry Neugebauer, ${ }^{3}$ B. T. Solfer, ${ }^{3,4}$ David T. Frayer, ${ }^{4}$ and James J. Condon ${ }^{5}$ \\ Received 2001 June 3 ; accepted 2001 July 9
}

\begin{abstract}
New infrared and millimeter observations from Keck, Palomar, ISO, and OVRO and archival data from the NRAO $^{6}$ VLA and IRAS are presented for the low-metallicity blue compact dwarf galaxy SBS $0335-052$. Mid-infrared imaging shows this young star-forming system is compact $(0.31 ; 80 \mathrm{pc})$ at $12.5 \mu \mathrm{m}$. The large $\mathrm{Br} \gamma$ equivalent width $(235 \AA)$ measured from integral field spectroscopy is indicative of a $\sim 5$ Myr starburst. The central source appears to be optically thin in emission, containing both a warm $(\sim 80 \mathrm{~K})$ and a hot $(\sim 210 \mathrm{~K})$ dust component, and the overall interstellar radiation field is quite intense, about 10,000 times the intensity in the solar neighborhood. CO emission is not detected, though the galaxy shows an extremely high global $\mathrm{H}$ I gas-to-dust mass ratio, high even for blue compact dwarfs. Finally, the galaxy's ratio of mid-infrared-to-optical and mid-to-near-infrared luminosities are quite high, whereas its far-infrared-to-radio and far-infrared-to-optical flux ratios are surprisingly similar to what is seen in normal star-forming galaxies. The relatively high bolometric infrared-to-radio ratio is more easily understood in the context of such a young system with negligible nonthermal radio continuum emission. These new lines of evidence may outline features common to primordial galaxies found at high redshift.
\end{abstract}

Key words: galaxies: individual (SBS 0335-052) - galaxies: ISM

On-line material: color figures

\section{INTRODUCTION}

The blue compact dwarf galaxy SBS $0335-052$ is an extremely metal-poor galaxy $\left(Z_{\odot} / 41\right.$; Izotov et al. 1997), with only I $\mathrm{Zw} 18$ known to have a lower metal abundance. At a radial velocity $c z=4043 \mathrm{~km} \mathrm{~s}^{-1}$, SBS $0335-052$ is at a distance of $53 \mathrm{Mpc}$ for $H_{0}=75 \mathrm{~km} \mathrm{~s}^{-1} \mathrm{Mpc}^{-1}$ (1" corresponds to $258 \mathrm{pc}$ ). Recent observations have highlighted several interesting characteristics of SBS $0335-052$ and its fainter companion $80^{\prime \prime}(22 \mathrm{kpc})$ to the west. In addition to showing very low metallicity, the bright starburst region of SBS $0335-052$ is situated in an extensive $\mathrm{H}$ I envelope, has an extremely large $B r y$ equivalent width, and appears unexpectedly bright in the mid-infrared, with a $12 \mu \mathrm{m}$-to- $B$-band luminosity ratio greater than 2 (Pustilnik et al. 2001; Vanzi et al. 2000; Thuan, Sauvage, \& Madden 1999). The last property is particularly intriguing since the galaxy exhibits anemic levels of metals. Moreover, Thuan, Sauvage, \& Madden found that the mid-infrared spectrum lacks the standard mid-infrared dust emission features, is dominated by a strong continuum, and peaks at a surprisingly short wavelength, suggestive that the radiating dust is embedded

\footnotetext{
${ }^{1}$ Based in part on observations obtained at the W. M. Keck Observatory, which is operated as a scientific partnership among the California Institute of Technology, the University of California, and the National Aeronautics and Space Administration.

${ }^{2}$ Division of Physics, Mathematics and Astronomy, California Institute of Technology 320-47, Pasadena, CA 91125.

${ }^{3}$ Palomar Observatory, California Institute of Technology 320-47, Pasadena, CA 91125.

${ }^{4}$ SIRTF Science Center, California Institute of Technology 314-6, Pasadena, CA 91125.

${ }^{5}$ National Radio Astronomy Observatory, 520 Edgemont Road, Charlottesville, VA 22903.

${ }^{6}$ The National Radio Astronomy Observatory is a facility of the National Science Foundation operated under cooperative agreement by Associated Universities, Inc.
}

in an extremely intense interstellar radiation field. $H S T$ optical observations by Papaderos et al. (1998) indicate an underlying stellar population of mass $\sim 3 \times 10^{6} M_{\odot}$ and an age younger than $100 \mathrm{Myr}$. Near-infrared data on the handful of bright super star clusters that dominate the galaxy's optical luminosity suggest that the central starforming region is even younger, of order $5 \mathrm{Myr}$ (Vanzi et al. 2000).

The unusual mid-infrared properties of SBS 0335-052 merit high spatial resolution studies: is the opacity at midinfrared wavelengths consistent with $A_{V} \sim 20 \mathrm{mag}$ (Thuan et al. 1999) or $A_{V} \sim 0.6 \mathrm{mag}$ (Izotov et al. 1997)? Can the emission source be resolved? What fraction of the midinfrared light is associated with the brightest star clusters? For example, Soifer et al. (2000) and Soifer et al. (2001) find that nearly all of the luminous and ultraluminous infrared galaxies in their samples emit a substantial fraction $(>50 \%)$ of their mid-infrared luminosity in compact $<100-300 \mathrm{pc}-$ sized cores. Similar information on dwarf galaxies in general, and SBS 0335-052 in particular, does not exist; mid-infrared data from IRAS and ISO have an angular resolution of several to tens of arcseconds, precluding detailed studies of compact mid-infrared emission. In this paper we present diffraction-limited mid-infrared imaging using the Keck telescope to investigate the innermost regions of SBS 0335-052. Additional near-infrared integral field spectroscopy and far-infrared, radio, and millimeter data are presented to further constrain the energetics of the interstellar medium.

While the physics of the interstellar medium in lowmetallicity dwarf galaxies is interesting in its own right, this relatively nearby system allows us to study in detail a truly young galaxy and thus could be a useful template for understanding galaxy formation at high redshifts. This comparison would be especially relevant if normal galaxies are assembled from a collection of small systems (e.g., van 
Dokkum et al. 1999; Cole et al. 2000). For example, $25 \mu \mathrm{m}$ observations with NGST of $z \approx 2$ galaxies, some of which may be in their earliest stages of development, may be usefully compared to (similar rest wavelength) mid-infrared observations of SBS $0335-052$.

\section{OBSERVATIONS AND DATA REDUCTION}

\subsection{Keck Near- and Mid-Infrared Imaging}

The mid-infrared observations of SBS $0335-052$ were made using the imaging mode of the Long Wavelength Spectrograph (LWS; Jones \& Puetter 1993) at the $f / 25$ forward Cassegrain focus of the Keck I Telescope. The object was centered in the LWS field of view by first imaging in $K$ band with the Near-Infrared Camera (NIRC; Matthews \& Soifer 1994) and then offsetting the position of the brightest peak at $K$ to the center of the LWS field.

The principal observations were made on the night of 2000 December 7, although observations of variable quality were made on 1999 November 24, 2000 January 26, and 2000 September 10. The December and September runs were photometric. A chopper was set to a north-south chopping amplitude of $5^{\prime \prime}$ at a frequency $\sim 5 \mathrm{~Hz}$. The telescope was nodded $5^{\prime \prime}$ in the north-south direction. The data were reduced by differencing the two images obtained within the chop pairs at each nod location and then coadding the resulting positive images. Because of the chopper and telescope nod spacings employed for the observations, the flux outside a $5^{\prime \prime}$ diameter region is suppressed. The pixel scale is 0.08 pixel $^{-1}$ for the mid-infrared data and 0 .'15 pixel $^{-1}$ for the near-infrared data. No flat-field corrections were performed.

Photometric data were obtained at $12.5 \mu \mathrm{m}$ $(\Delta \lambda=1.0 \mu \mathrm{m})$. The photometry was calibrated based on observations of the bright stars HR $1457 \quad(\alpha$ Tau; $[12.5 \mu \mathrm{m}]=-3.07 \mathrm{mag})$ and $\operatorname{HR} 8775 \quad(\beta \quad \mathrm{Peg}$; $[12.5 \mu \mathrm{m}]=-2.55 \mathrm{mag}$ ) whose magnitudes, in turn, were based on IRAS photometry and previous Keck midinfrared photometry. The systematic uncertainties in the photometry, based on the internal consistency of the observations, is estimated to be $5 \%$. However, the absolute calibration of standard star magnitudes and the photometric zero points each night limit the overall photometric accuracy to $15 \%-20 \%$. The flux density corresponding to 0.0 mag was taken as $26.2 \mathrm{Jy}$, following the prescription given in the Explanatory Supplement to the IRAS Catalogs and Atlases (Beichman et al. 1985).

On the night of 2000 December 7, the total mid-infrared on-target observation time was 25 minutes. The observations of SBS $0335-052$ were interleaved with six observations of the star IRC-10 046 in order to determine a contemporaneous point spread function (PSF).

\subsection{Palomar Near-Infrared Integral Field Spectroscopy}

Near-infrared observations of the $\mathrm{Br} \gamma$ line were carried out on the night of 2000 October 14 using the Palomar Integral Field Spectrograph (PIFS; Murphy, Matthews, \& Soifer 1999) on the Palomar 200 inch Telescope. PIFS provides a 5".4 × 9".6 field of view using eight separate 0".67 $\times 9$ ".6 slits to feed two independent spectrographs within the same liquid $\mathrm{N}_{2}$-cooled Dewar. Each slit is four pixels wide and 58 pixels long $\left(0^{\prime \prime} 167 \mathrm{pixel}^{-1}\right)$. The highresolution mode was used, providing a spectral resolution of $\lambda / \Delta \lambda \sim 1450\left(\Delta v \sim 200 \mathrm{~km} \mathrm{~s}^{-1}\right)$ near the observed center wavelength of $2.195 \mu \mathrm{m}$.
The PIFS observations consisted of a set of eight 5 minute on-off integrations. Thus, 40 minutes were spent integrating on source, and an equal amount of time off source (using a $30^{\prime \prime}$ nod to the north). For these observations, the $5^{\prime \prime} .4 \times 99^{\prime \prime} .6$ field of view was oriented with the long axis at a position angle of $90^{\circ}$ (the effect of differential atmospheric refraction is minimal for integral field spectroscopy). A positional dithering pattern was employed for the sequence of observations to recover seeing-limited spatial sampling in the cross-slit direction. Spectral calibration lamp data were taken immediately afterward; wavelength calibration is provided through a combination of these data and the available $\mathrm{OH}$ airglow lines (Oliva \& Origlia 1992). The observations were taken under photometric conditions. Spectrophotometric calibration was performed via spectral imaging of the Persson et al. (1998) standard star 9101, thereby establishing the continuum flux density at $2.195 \mu \mathrm{m}$; the $2.195 \mu \mathrm{m}$ flux density of the standard star was estimated from an extrapolation of its $K s$ band $\left(\lambda_{\mathrm{c}}=\right.$ $2.157 \mu \mathrm{m})$ and $K$-band $\left(\lambda_{\mathrm{c}}=2.179 \mu \mathrm{m}\right)$ flux density trend. Corrections for atmospheric opacity and spectral flatfielding are derived from observations of HR 1232, a 5.8 mag G9 V star. Details of the calibration technique and the PIFS data reduction process are given in Murphy et al. (2000).

An estimate of the near-infrared PSF was obtained after the first 20 minutes of on-source integration and after the second 20 minutes of on-source integration, with each measurement comprised of four dithered $10 \mathrm{~s}$ exposures in $K$ band of a nearby field star using the PIFS imaging mode. An azimuthally averaged radial profile of the PSF target in the first case yields a full width at half-maximum (FWHM) of approximately 0 ". 8 . After refocusing, our second measurement was $\mathrm{FWHM} \approx 0.5$, indicating that the observations were performed in generally good seeing conditions.

\subsection{Owens Valley $\mathrm{CO}$ Maps}

SBS 0335-052 was observed using the Owens Valley Millimeter Array between February and May of 1999. The source was observed in two configurations of the six $10.4 \mathrm{~m}$ telescopes with baselines ranging from 15 to $119 \mathrm{~m}$. The lines ${ }^{12} \mathrm{CO}(J=1 \rightarrow 0)$ and ${ }^{12} \mathrm{CO}(J=2 \rightarrow 1)$ were simultaneously observed assuming a redshift of $4076 \mathrm{~km} \mathrm{~s}^{-1}$ (Thuan, Izotov, \& Lipovetsky 1997). The CO(1-0) data were recorded using a digital correlator configured with $64 \times 2 \mathrm{MHz}$ channels centered on $113.2750 \mathrm{GHz}$, while the $\mathrm{CO}(2-1)$ line was observed with $59 \times 4 \mathrm{MHz}$ channels centered on $227.4456 \mathrm{GHz}$. Both spectrometer setups provided approximately $300 \mathrm{~km} \mathrm{~s}^{-1}$ of bandwidth coverage, which is more than adequate for SBS $0335-052$. In addition to the spectral line data, the $3 \mathrm{~mm}$ continuum and $1 \mathrm{~mm}$ continuum were observed with a $1 \mathrm{GHz}$ bandwidth for both the lower and upper sidebands. Typical single-sideband system temperatures were approximately $800 \mathrm{~K}$, corrected for telescope losses and the atmosphere. The nearby quasar PKS B0420-014 was observed every 20 minutes for gain and phase calibration. Absolute flux calibration was determined from observations of Uranus, Neptune, and 3C 273. The absolute calibration uncertainty for the data is approximately $15 \%$ at $3 \mathrm{~mm}$ and $25 \%$ at $1 \mathrm{~mm}$. After data editing, we obtained effectively $20.6 \mathrm{hr}$ of on-source integration time at $3 \mathrm{~mm}$ and $6.6 \mathrm{hr}$ of good integration time at $1 \mathrm{~mm}$. The beam sizes computed using natural weighting to minimize the noise are 5". $\times$ 3".8 and 2". $\times$ 2".0 for the $113.2750 \mathrm{GHz}$ 
and the $227.4456 \mathrm{GHz}$ maps, respectively, providing spatial resolution of $1.4 \times 1.0 \mathrm{kpc}$ and $750 \times 520 \mathrm{pc}$.

\subsection{Very Large Array Radio Continuum Maps}

VLA archival data are available at $1.49 \mathrm{GHz}$ from the B configuration. The data processing includes the primarybeam correction and results in an image with a 6" FWHM Gaussian beam and a rms noise level of $0.13 \mathrm{mJy}^{\text {beam }}{ }^{-1}$. The point source sensitivity is about a factor of 3 better than the NRAO VLA Sky Survey (NVSS; Condon et al. 1998), but the surface brightness sensitivity is about 20 times poorer (the NVSS has a 45" FWHM beam).

\subsection{Infrared Space Observatory Far-Infrared Photometry}

Results from ISOPHOT observations were kindly provided by M. Sauvage. SBS $0335-052$ was detected with the PHT-C C100 camera system and the $50 \mu \mathrm{m}$ filter $\left(\lambda_{\text {ref }}=65\right.$ $\mu \mathrm{m}, \lambda_{\text {cen }}=67.3 \mu \mathrm{m}, \Delta \lambda=57.8 \mu \mathrm{m}$ ), with a far-infrared flux density of $f_{v}(67 \mu \mathrm{m})=112 \mathrm{mJy}$ for $\mathrm{S} / \mathrm{N} \approx 4$.

\subsection{Data from the Infrared Astronomical Satellite}

SBS $0335-052$ was not detected by IRAS, but robust estimates of the $12,25,60$, and $100 \mu \mathrm{m}$ rms levels can be estimated from the (7-10) separate scans made by $I R A S$ at this location. The SCANPI software has been used to determine the median rms levels.

\section{RESULTS}

\subsection{Mid-Infrared Properties}

The $12.5 \mu \mathrm{m}$ image of SBS $0335-052$ is presented in Figure 1. From the Keck observations the $12.5 \mu \mathrm{m}$ magnitude of SBS $0335-052$ is $7.2 \pm 0.2 \mathrm{mag}$, which corresponds to $35 \pm 6 \mathrm{mJy} \quad(6 \%$ statistical and $15 \%$ systematic uncertainties). The statistical uncertainty is the standard deviation based on six differences from the average value.

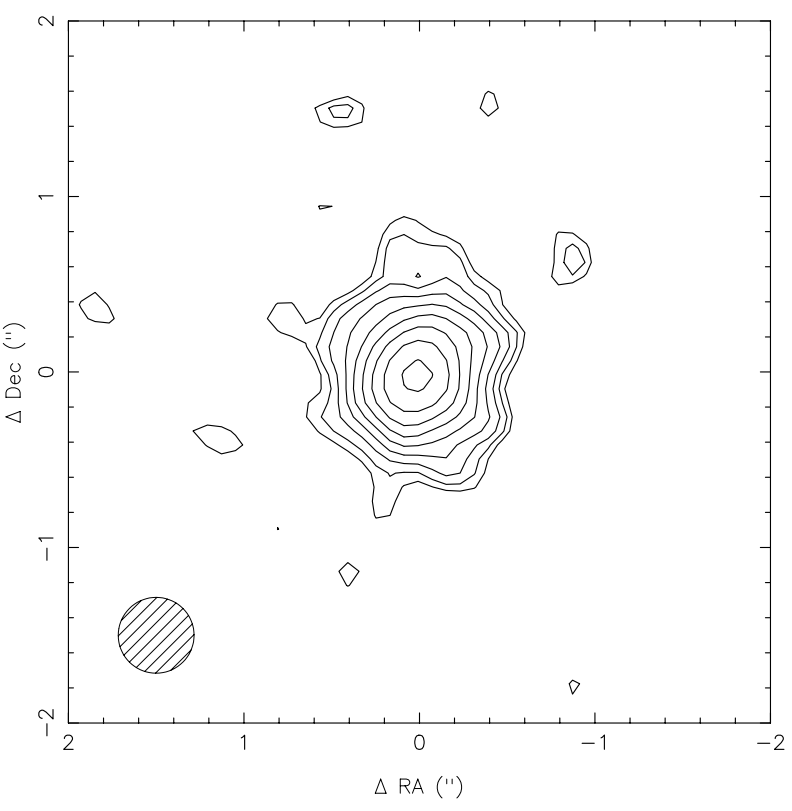

FIG. 1.-Keck I $12.5 \mu \mathrm{m}$ image of SBS $0335-052$, taken on the night of 2000 December. The contours are scaled by factors of 1.342 , with the highest and third-highest contours enclosing regions where the emission is greater than $90 \%$ and $50 \%$ of the peak flux, respectively; the lowest contour is at the $3 \sigma$ level. The FWHM of the PSF is displayed in the lower left-hand corner. North is up, east is to the left, and the central coordinates are those given in Table 1.
This flux density over a bandpass spanning approximately 12.0 to $13.0 \mu \mathrm{m}$ is consistent with the $I S O$ mid-infrared data obtained by Thuan et al. (1999), from which they estimate $f_{v}(12 \mu \mathrm{m}) \simeq 21 \pm 6 \mathrm{mJy}$ and $f_{v}(14.9 \mu \mathrm{m}) \simeq 35 \pm 11 \mathrm{mJy}$ from ISOCAM photometry using the LW10 $(\Delta \lambda=7.0 \mu \mathrm{m})$ and LW9 $(\Delta \lambda=2.0 \mu \mathrm{m})$ filters, respectively $(30 \%$ uncertainties have been attached to the $I S O$ fluxes, reflecting the typical calibration uncertainty in such data). Moreover, the ISOCAM CVF spectrum suggests a $12.5 \mu \mathrm{m}$ flux level near $30 \mathrm{mJy}$. At a distance of $53 \mathrm{Mpc}$, the mid-infrared luminosity of SBS $0335-052$ is $v L_{v}(12.5 \mu \mathrm{m}) \equiv L(12.5 \mu \mathrm{m})=$ $7.5 \times 10^{8} L_{\odot}$ and the far-infrared luminosity is $L(67 \mu \mathrm{m})=$ $4.4 \times 10^{8} L_{\odot}$. Table 1 summarizes the main results and general properties of SBS $0335-052$.

As pointed out by Thuan et al. (1999), the IRASequivalent $12 \mu \mathrm{m}$-to-blue ratio for SBS 0335-052 is several times greater than found for spiral galaxies and more than twice as large as the typical value for blue compact dwarf galaxies. The mean ratio for the Thuan \& Martin (1981) catalog of 115 blue compact dwarfs is $\langle\log L(12 \mu \mathrm{m}) / L(0.44 \mu \mathrm{m})\rangle=-0.12 \pm 0.27$, and $\langle\log L(12$ $\mu \mathrm{m}) / L(0.44 \mu \mathrm{m})\rangle=-0.55 \pm 0.06$ for the 69 normal starforming galaxies described in Helou et al. (1996) and Dale et al. (2000), whereas SBS 0335-052 shows $\log L(12.5 \mu \mathrm{m}) /$ $L(0.44 \mu \mathrm{m}) \sim 0.25$. The mid-to-near-infrared and mid-tofar-infrared luminosity ratios are likewise elevated: $\log L(12.5 \mu \mathrm{m}) / L(2.2 \mu \mathrm{m}) \simeq 1.2$ and $\log L(12.5 \mu \mathrm{m}) / L(67$ $\mu \mathrm{m}) \simeq 0.23$, high compared with the average values $\langle\log L(12 \mu \mathrm{m}) / L(2.2 \mu \mathrm{m})\rangle=-0.16 \pm 0.06$ and $\langle\log L(12$ $\mu \mathrm{m}) / L(60 \mu \mathrm{m})\rangle=-0.63 \pm 0.04$ for normal star-forming galaxies (Dale et al. 2000). Thus, in spite of the galaxy's depressed metallicity, hot dust is one of the energetically dominant features of its interstellar medium.

The $12.5 \mu \mathrm{m}$ source is compact (see Fig. 1), and the morphology at this wavelength is consistent with a circularly symmetric distribution of warm dust. The average observed FWHM of SBS $0335-052$ was $0.48 \pm 0.02$, whereas the average FWHM of seven separate PSF observations was $0.37 \pm 0.01$. Thus, we conclude that SBS 0335-052 is slightly resolved; the intrinsic FWHM after deconvolution of the individual PSFs is $0.31 \pm 0.02(80 \pm 5 \mathrm{pc})$. The uncertainty is statistical and is based on six differences from the FWHM of the PSF. A Richardson-Lucy deconvolution (Lucy 1974) gives a slightly smaller intrinsic size and likewise shows no significant departures from azimuthal symmetry. The Keck Telescope and LWS do not provide absolute positions to an accuracy of less than 1", and so two independent measurements were made to give the position of the $12.5 \mu \mathrm{m}$ image relative to the images at other wavelengths. NIRC shares a common focal plane with LWS, and it is possible to accurately move the telescope from NIRC to LWS. At $2.2 \mu \mathrm{m}$ SBS $0335-052$ is dominated by a bright nucleus; measurements of SBS $0335-052$ at $12.5 \mu \mathrm{m}$ were thus made by offsetting the telescope from this bright nucleus as seen at $2.2 \mu \mathrm{m}$ to the LWS array. To the $\sim \pm 0$ ".5 accuracy of these offsets, the compact source at $12.5 \mu \mathrm{m}$ coincides with the bright peak at $2.2 \mu \mathrm{m}$. The second relative position measurement is described below.

\subsection{Near-Infrared Integral Field Spectral Data}

The PIFS observations presented in Figure 2 show that the $\mathrm{Br} \gamma$ emission is apparently spatially extended: the line emission spans 1" FWHM (260 pc) compared with the 0 ".5-0"8 seeing at that wavelength. Whereas the signal-to- 
TABLE 1

DATA FOR SBS 0335-052

\begin{tabular}{|c|c|c|}
\hline Parameter & Value & Source \\
\hline Optical position (J2000) ...... & $033744.0,-050238$ & NED \\
\hline$c z_{\odot}\left(\mathrm{km} \mathrm{s}^{-1}\right) \ldots \ldots \ldots \ldots \ldots \ldots$ & 4043 & NED \\
\hline Distance $^{\mathrm{a}}(\mathrm{Mpc}) \ldots \ldots \ldots \ldots \ldots$ & 53.3 & \\
\hline$m_{B}^{\mathrm{b}}(\mathrm{mag}) \ldots \ldots \ldots \ldots \ldots \ldots \ldots$ & 16.76 & Papaderos et al. 1998 \\
\hline$m_{K}{ }^{\mathrm{b}}(\mathrm{mag}) \ldots \ldots \ldots \ldots \ldots \ldots \ldots$ & 15.43 & Vanzi et al. 2000 \\
\hline$f_{v}(0.44 \mu \mathrm{m})^{\mathrm{c}}(\mathrm{mJy}) \ldots \ldots \ldots \ldots$ & $0.84 \pm 0.02$ & Papaderos et al. 1998 \\
\hline$f_{v}(2.2 \mu \mathrm{m})^{\mathrm{c}}(\mathrm{mJy}) \ldots \ldots$ & $0.42 \pm 0.01$ & Vanzi et al. 2000 \\
\hline$f_{v}(12.5 \mu \mathrm{m})(\mathrm{mJy}) \ldots \ldots$. & $35 \pm 6$ & This work \\
\hline$f_{v}(67.3 \mu \mathrm{m})(\mathrm{mJy}) \ldots \ldots \ldots \ldots$ & $112 \pm 28$ & This work \\
\hline$f(\mathrm{Br} \gamma)\left(\mathrm{W} \mathrm{m}^{-2}\right) \ldots \ldots \ldots \ldots \ldots$ & $6 \times 10^{-18}$ & This work \\
\hline$f_{v}(12 \mu \mathrm{m})^{\mathrm{d}}(\mathrm{mJy}) \ldots \ldots \ldots$ & $<72$ & $I R A S$ \\
\hline$f_{v}(25 \mu \mathrm{m})^{\mathrm{d}}(\mathrm{mJy}) \ldots \ldots$ & $<117$ & $I R A S$ \\
\hline$f_{v}(60 \mu \mathrm{m})^{\mathrm{d}}(\mathrm{mJy}) \ldots \ldots \ldots \ldots \ldots$ & $<165$ & $I R A S$ \\
\hline$f_{v}(100 \mu \mathrm{m})^{\mathrm{d}}(\mathrm{mJy}) \ldots \ldots \ldots \ldots . .$. & $<210$ & $I R A S$ \\
\hline$S_{\mathrm{CO}(1-0)} \mathrm{d}^{\mathrm{d}}\left(\mathrm{Jy} \mathrm{km} \mathrm{s}^{-1}\right) \ldots \ldots \ldots$ & $<1.2$ & This work \\
\hline$S_{\mathrm{CO}(2-1)} \mathrm{d}\left(\mathrm{Jy} \mathrm{km} \mathrm{s}^{-1}\right) \ldots \ldots \ldots$ & $<3.0$ & This work \\
\hline$f_{v}(1.49 \mathrm{GHz})(\mathrm{mJy}) \ldots \ldots \ldots \ldots$ & $0.4 \pm 0.1$ & This work \\
\hline$L(0.44 \mu \mathrm{m})^{\mathrm{e}}\left(L_{\odot}\right) \ldots \ldots \ldots \ldots \ldots$ & $4.3 \times 10^{8}$ & Papaderos et al. 1998 \\
\hline$L(12.5 \mu \mathrm{m})^{\mathrm{e}}\left(L_{\odot}\right) \ldots \ldots \ldots \ldots \ldots$ & $7.5 \times 10^{8}$ & This work \\
\hline$L(67.3 \mu \mathrm{m})^{\mathrm{e}}\left(L_{\odot}\right) \ldots \ldots \ldots \ldots \ldots$ & $4.4 \times 10^{8}$ & This work \\
\hline$M\left(\mathrm{H}_{2}\right)^{\mathrm{f}}\left(M_{\odot}\right) \ldots \ldots \ldots \ldots \ldots \ldots$ & $<4.1 \times 10^{7}$ & This work \\
\hline$M\left(\mathrm{H}_{2}\right)^{\mathrm{g}}\left(M_{\odot}\right) \ldots \ldots \ldots \ldots \ldots \ldots$ & $<4.9 \times 10^{8}$ & This work \\
\hline
\end{tabular}

NoTE.-Units of right ascension are hours, minutes, and seconds, and units of declination are degrees, arcminutes, and arcseconds.

${ }^{\text {a }}$ Computed in the Local Group reference frame and assuming $H_{0}=75 \mathrm{~km} \mathrm{~s}^{-1}$ $\mathrm{Mpc}^{-1}$.

b Corrected for Galactic extinction (Schlegel, Finkbeiner, \& Davis 1998).

${ }^{\mathrm{c}}$ Computed assuming $f_{v}(0.44 \mu \mathrm{m})=4260 \times 10^{-0.4 m_{B}} \mathrm{Jy}$ and $f_{v}(2.2 \mu \mathrm{m})=620$

$\times 10^{-0.4 m_{\mathrm{K}}}$ Jy (Bessel 1979).

d $3 \sigma$ upper limit.

${ }^{\mathrm{e}} L \equiv 4 \pi d^{2} v f_{v}$, where $v f_{v}$ is the power per octave. The solar luminosity is taken to be $L_{\odot}=3.826 \times 10^{26} \mathrm{~W}$.

${ }^{f}$ Computed assuming a Galactic $\mathrm{CO}$ to $\mathrm{H}_{2}$ conversion factor.

${ }^{g}$ Computed assuming a $\mathrm{CO}$ to $\mathrm{H}_{2}$ conversion factor equal to 12 times the Galactic value.

noise ratio of the PIFS continuum data is too low for us to accurately gauge by eye the center of the continuum distribution, a central moment analysis of the continuum and line emission maps yields a negligible $(<0.03 ;<10 \mathrm{pc})$ continuum-line offset; there is no evidence in the integral field spectroscopy data for the $60 \mathrm{pc}$ offset between the $\mathrm{Br} \gamma$ and $2 \mu \mathrm{m}$ continuum peaks claimed by Vanzi et al. (2000). By the design of the PIFS (Murphy et al. 1999), the Br $\gamma$ and $2.2 \mu \mathrm{m}$ continuum images are spatially tied together. If the ad hoc assumption is made that the $12.5 \mu \mathrm{m}$ emission coincides with the $\mathrm{Br} \gamma$ emission, this ties the $12.5 \mu \mathrm{m}$ emission and the $2.2 \mu \mathrm{m}$ continuum emission of SBS 0335-052 together. Figure 3 illustrates this assumption and thus orients the $12.5 \mu \mathrm{m}$ emission of SBS $0335-052$ relative to that at $\mathrm{Br} \gamma$ and $2.2 \mu \mathrm{m}$. Both this and the offsetting measurements previously described are consistent with the $12.5 \mu \mathrm{m}$ emission of SBS $0335-052$ coinciding with the bright peak in the $2.2 \mu \mathrm{m}$ continuum. Using 0 ".33 resolution $K s$ imaging, Vanzi et al. (2000) note a $\sim 1.5$ separation between two distinct components along the southeastnorthwest direction, and HST WFPC2 $V$ and $I$ imaging show several distinct clumps spanning $\sim 4^{\prime \prime}$ along a southeast-northwest orientation (Papaderos et al. 1998). Such substructure is seen in the Keck NIRC image, but the PIFS near-infrared data give only a faint hint of the multiple peaks, presumably due to the limited spatial resolution and sensitivity of the integral field spectroscopy data.
No velocity structure is resolved for the $\mathrm{Br} \gamma$ emission. This is not surprising since the resolution is of order $200 \mathrm{~km} \mathrm{~s}^{-1}$ and the VLA H I data of Pustilnik et al. (2001) only show $\sim 40 \mathrm{~km} \mathrm{~s}^{-1}$ solid-body rotation for the eastern $\mathrm{H}$ I peak that is centered on SBS 0335-052. Furthermore, the lack of significant velocity structure of the $\mathrm{Br} \gamma$ line is consistent with the small velocity dispersion $\left(<10 \mathrm{~km} \mathrm{~s}^{-1}\right)$ of the optical emission lines (Izotov et al. 1997).

Measures of the starburst timescale can be inferred from near-infrared continuum data. However, Vanzi et al. (2000) find that the combined helium, $\mathrm{H}_{2}$, and $\mathrm{Br} \gamma$ emission lines contribute about $50 \%$ of the $K$-band flux (within their $1^{\prime \prime}$ $\times 0$ " 8 spectroscopy slit) and that $\mathrm{Br} \gamma$ alone accounts for $6 \%-7 \%$. To within $10 \%$ certainty, the integrated $\mathrm{Br} y$ flux from the PIFS data is $6 \times 10^{-18} \mathrm{~W} \mathrm{~m}^{-2}$, or approximately $4 \%$ of the global $K$-band flux. The $\mathrm{Br} \gamma$ equivalent width is also indicative of the starburst age (\$ 4.3) and is not contaminated by other nebular emission lines. From the PIFS data the $\mathrm{Br} \gamma$ equivalent width is $235 \pm 33 \AA$. A cursory review of the literature did not find a larger extragalactic Bry equivalent width.

\subsection{Radio Continuum Results}

SBS $0335-052$ is unresolved in the high-resolution map $\left(\mathrm{FWHM}=6^{\prime \prime}\right.$ beam) at $1.49 \mathrm{GHz}$ (Fig. 4). There is a $3 \sigma$ source with a peak brightness of $0.40 \mathrm{mJy}^{\text {beam }}{ }^{-1}$ at the nominal optical position of SBS $0335-052$; the radio 

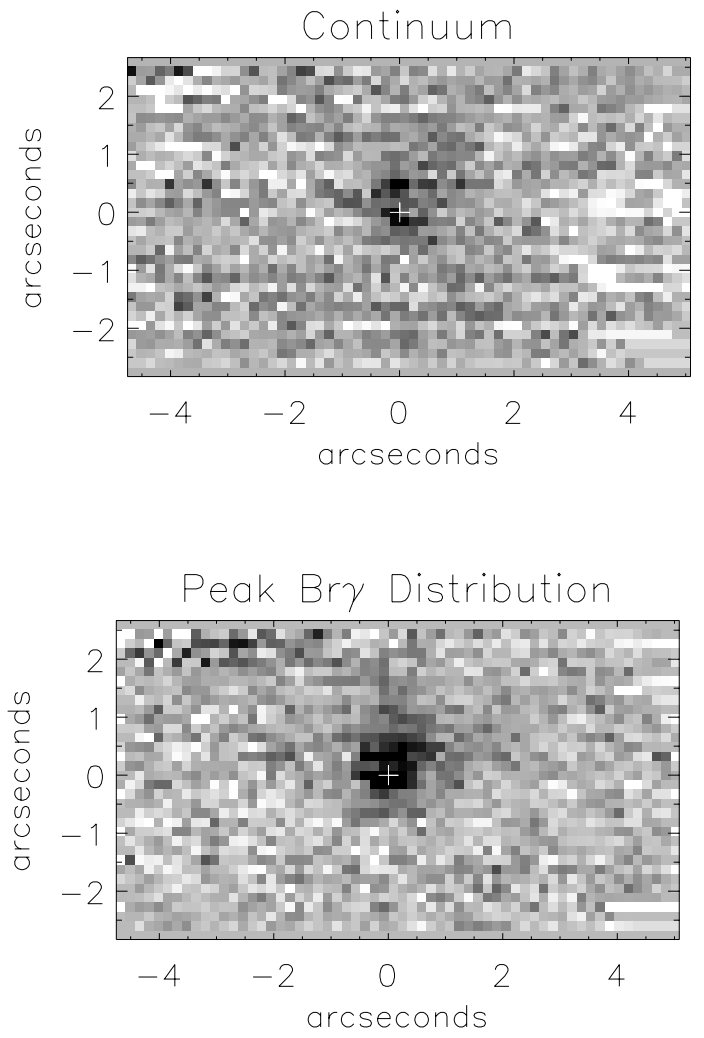
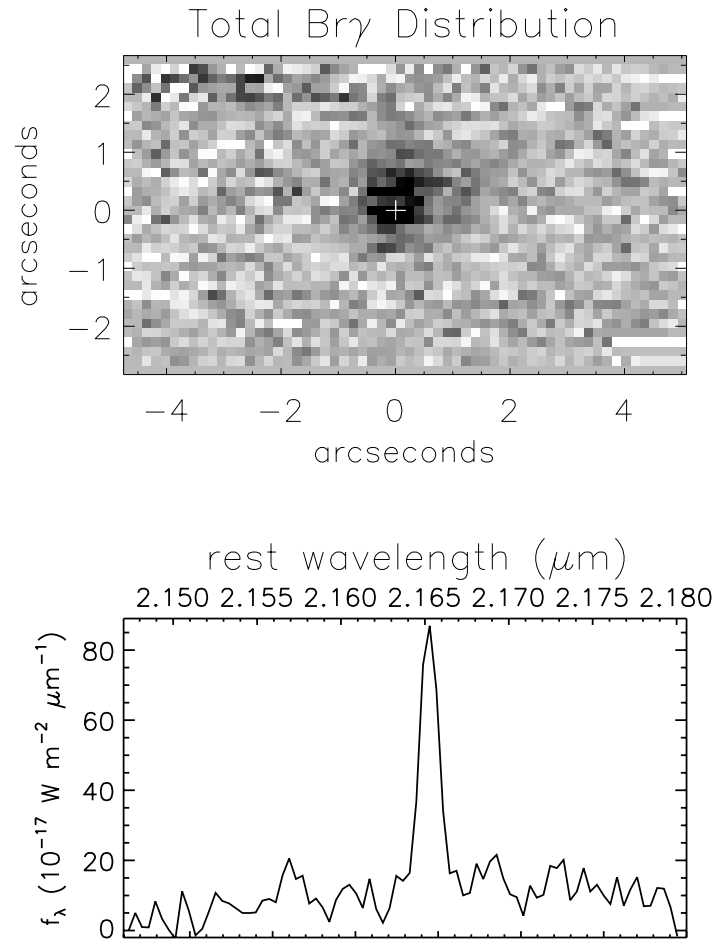

2.1802 .1852 .1902 .1952 .2002 .2052 .210

observed wavelength ( $\mu \mathrm{m})$

FIG. 2.-PIFS Bry data for SBS 0335-052. At top left is the line-free continuum image reconstructed from the data cube. At top right is the total Bry emission, continuum subtracted, summed from -170 to $+170 \mathrm{~km} \mathrm{~s}^{-1}$ relative to the systemic velocity. A slightly higher contrast image is shown at lower left, constructed from a single spectral resolution element centered for each spatial pixel on the wavelength of maximum line emission intensity. In all images, the continuum peak location is indicated by a cross, and the central coordinates are those given in Table 1. Finally, the near-infrared spectrum containing the $\mathrm{Br} \gamma$ line is displayed at lower right.

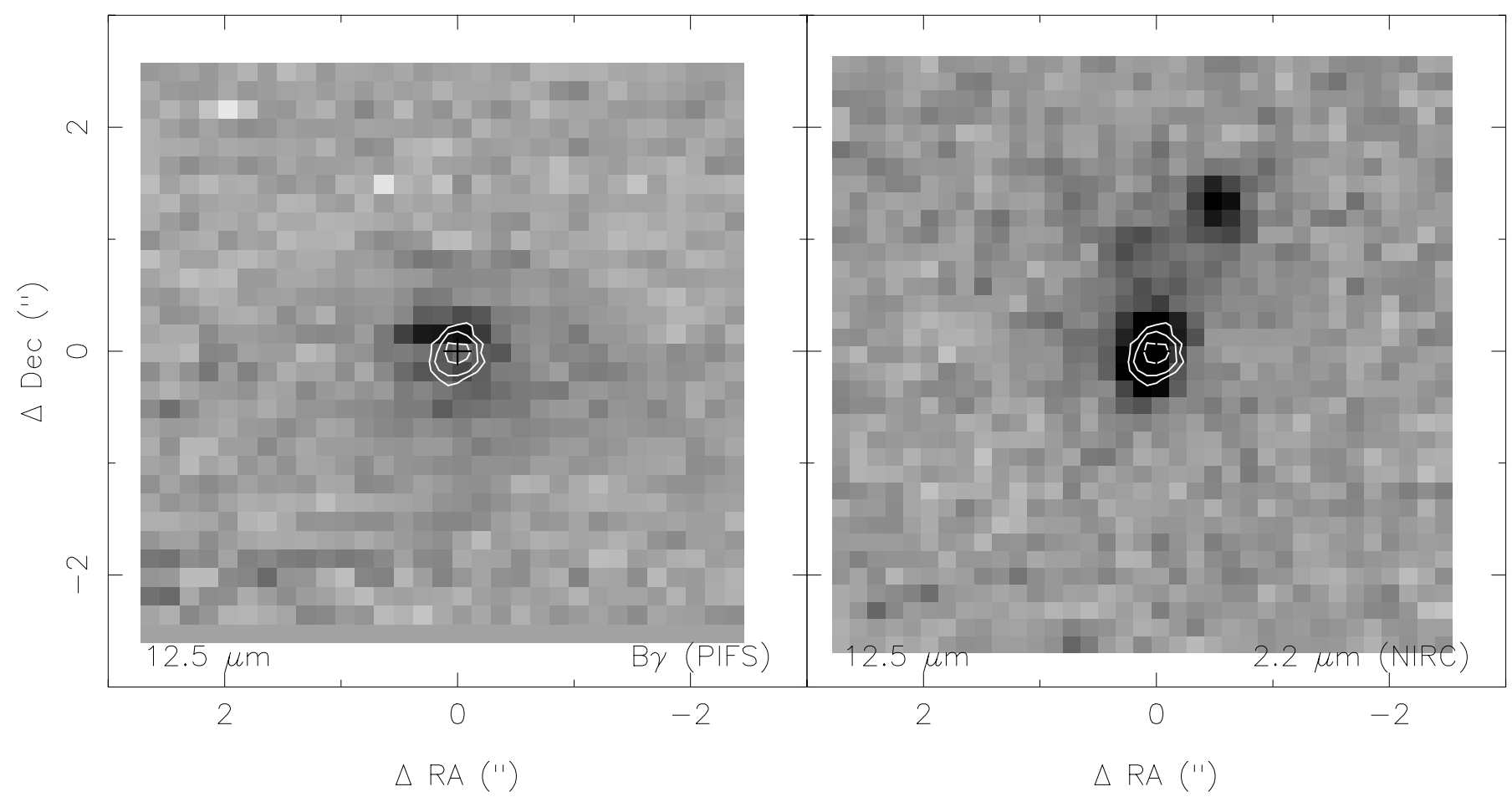

FIG. 3.-The Keck $12.5 \mu$ m contours enclosing regions where the emission is greater than $90 \%, 67 \%$, and $50 \%$ of the peak flux overlaid on the PIFS Br $\gamma$ data and the Keck NIRC $2.2 \mu \mathrm{m}$ continuum data. The $2.2 \mu \mathrm{m}$ emission contains multiple unresolved components that presumably correspond to the super star clusters described in Thuan et al. (1997). Table 2 provides a comparison of the size of SBS $0335-052$ at different wavelengths. To $~ 0$ ". 5 accuracy, the peak of the $12.5 \mu \mathrm{m}$ data is aligned with the brightest peak of the $2.2 \mu \mathrm{m}$ continuum and Br $\gamma$ emission (see the associated discussion in the text). Both frames are oriented with north up and east to the left, and the central coordinates are those given in Table 1. 


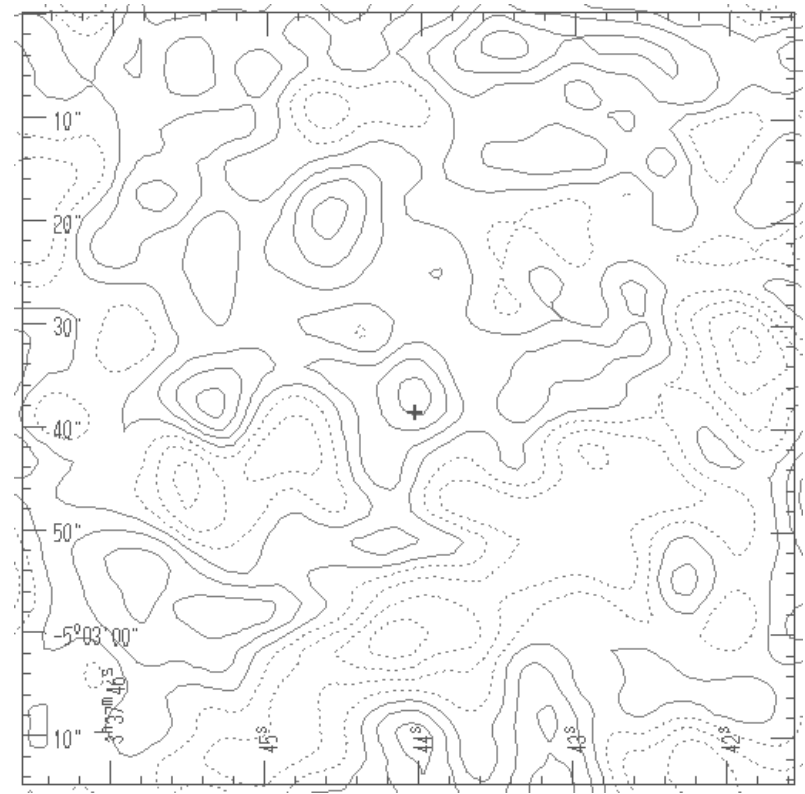

FIG. 4. $-1.49 \mathrm{GHz}$ data from the VLA archive. The $3 \sigma$ source located at the center of this field is at the nominal optical position of SBS $0335-052$, which is indicated by a cross. The contour levels are plotted at $0.15 \mathrm{mJy}^{\text {beam }}{ }^{-1}$ intervals, starting at $0.45 \mathrm{mJy}^{\text {beam }}{ }^{-1}$. Coordinates are given in the epoch $\mathbf{J} 2000.0$.

source has a flux density of $0.40 \mathrm{mJy}$. Each coordinate has a rms error of about $1^{\prime \prime}$ due to noise, but this is much better than the NVSS positional uncertainty of $\sim 7^{\prime \prime}$ for faint sources like SBS $0335-052$. No extended radio continuum emission is visible, but a $2.3 \pm 0.4 \mathrm{mJy}$ source (the NVSS total flux) smooth source $30^{\prime \prime}$ in size would have a brightness of only $0.1 \mathrm{mJy}$ beam $^{-1}$ and be completely undetectable. Indeed, no $20 \mathrm{~cm}$ continuum emission is detected in a VLA survey with $1 \mathrm{mJy}$ beam $^{-1}(4 \sigma)$ sensitivity and a $20^{\prime \prime} 5 \times 14^{\prime \prime}$ beam (Pustilnik et al. 2001). On the other hand, the offset of the NVSS source from the optical position (12" at a position angle of $50^{\circ}$ east of north) is large enough that the NVSS source may well be a blend of SBS 0335-052 and two other faint sources to the northeast visible in the contour plot. In short, the flux of the nearby NVSS source probably overestimates the total flux of SBS 0335-052, but the $0.40 \mathrm{mJy}$ component probably corresponds to the compact part of SBS $0335-052$, particularly since they agree well in position. The chance that this is a nondetection and is instead a random $3 \sigma$ noise bump coincidentally close to the nominal position of SBS $0335-052$ is low. The probability of finding an unrelated radio source with flux $\geq 0.4 \mathrm{mJy}$ within $6^{\prime \prime}$ is less than $2 \times 10^{-3}$ (Langston et al. 1990).

\subsection{CO Results}

As seen in Figure 5, neither CO nor millimeter continuum emission was detected. The rms limits of 12 and $30 \mathrm{mJy}$ were achieved for the $\mathrm{CO}(1-0)$ and $\mathrm{CO}(2-1)$ observations, respectively, from an average over four spectral channels (Fig. 5). No significant emission or absorption features were detected anywhere in the field. Since the synthesized beams are larger than the bright central starburst regions, it is not expected that the interferometer has resolved out the $\mathrm{CO}$ emission. Given that the $\mathrm{CO}$ line widths are typically only half the $\mathrm{H}$ I line widths in metal-poor

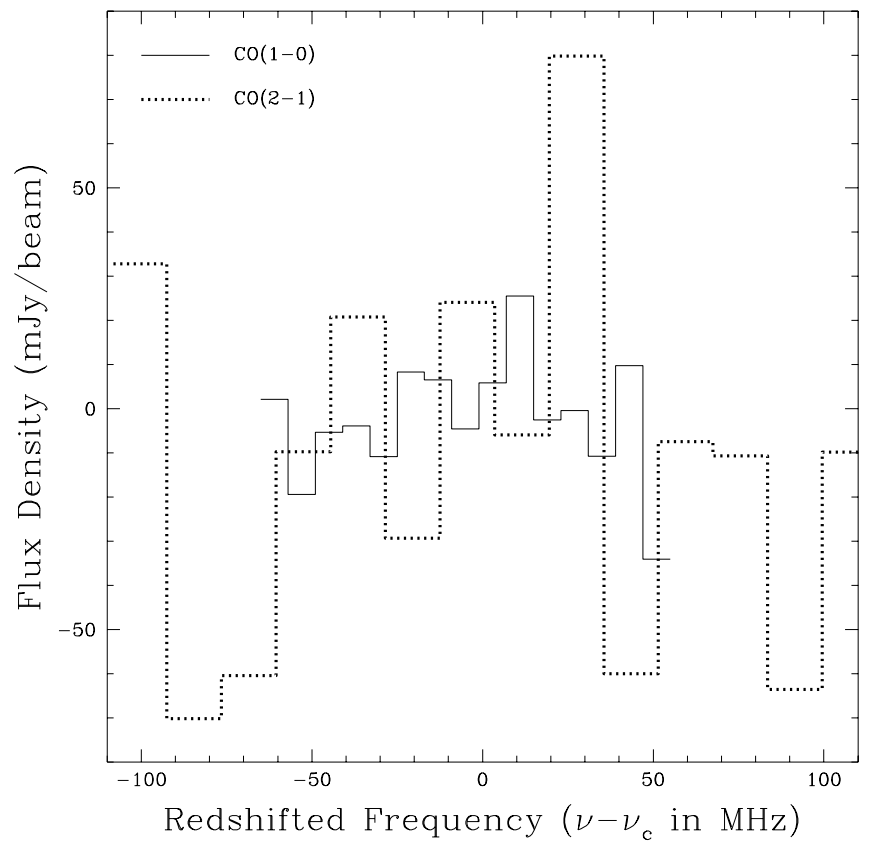

FIG. 5.-The $\mathrm{CO}(1-0)$ and $\mathrm{CO}(2-1)$ spectra, centered at $v_{\mathrm{c}}=113.73$ $\mathrm{GHz}$ and $v_{\mathrm{c}}=227.44 \mathrm{GHz}$, respectively. The observations assumed an optical redshift of $4076 \mathrm{~km} \mathrm{~s}^{-1}$ in the Local Group reference frame (Thuan et al. 1997). The rms level for the (1-0) data is $12 \mathrm{mJy}$, and $30 \mathrm{mJy}$ for the (2-1) line.

galaxies (Sage et al. 1992), a CO line width of approximately $50 \mathrm{~km} \mathrm{~s}^{-1}$ would be expected for SBS $0335-052$. With this adopted line width, the $3 \sigma$ upper limits are $S_{\mathrm{CO}(1-0)}<1.2$ $\mathrm{Jy} \mathrm{km} \mathrm{s}{ }^{-1}$ and $S_{\mathrm{CO}(2-1)}<3.0 \mathrm{Jy} \mathrm{km} \mathrm{s}{ }^{-1}$ for the integrated $\mathrm{CO}$ line fluxes. For the continuum data, the $1 \mathrm{GHz}$ bandwidths in both the upper and lower sidebands were averaged, resulting in $3 \sigma$ upper limits of $f_{v}(112 \mathrm{GHz})<2.3 \mathrm{mJy}$ and $f_{v}(229 \mathrm{GHz})<11 \mathrm{mJy}$.

Given the observational limits, the $\mathrm{CO}(1-0)$ data provide a more stringent constraint on the molecular hydrogen gas mass than the $\mathrm{CO}(2-1)$ data-blue compact galaxies typically have brightness temperature ratios of $T_{b}(2-1) / T_{b}(1-0)=0.3-0.6$ (Sage et al. 1992). We thus concentrate on the $\mathrm{CO}(1-0)$ data for the remainder of this section. The integrated $\mathrm{CO}(1-0)$ flux in $\mathrm{Jy} \mathrm{km} \mathrm{s}^{-1}$ is related to the molecular hydrogen gas mass by $M\left(\mathrm{H}_{2}\right)=1.2$ $\times 10^{4} S_{\mathrm{CO}(1-0)}(d / \mathrm{Mpc})^{2}\left(\alpha / \alpha_{\mathrm{G}}\right) M_{\odot}$, where $\alpha_{\mathrm{G}}$ is the Galactic $\mathrm{CO}$ to $\mathrm{H}_{2}$ conversion factor (Wilson 1995). Adopting the Galactic conversion factor results in $M\left(\mathrm{H}_{2}\right)<4.1 \times 10^{7}$ $M_{\odot}(3 \sigma)$. However, the Galactic conversion factor is thought to significantly underestimate the molecular hydrogen gas mass in the metal-poor galaxies. Estimating the proper value of $\alpha$ for SBS $0335-052$ is somewhat problematic since $\mathrm{CO}$ has yet to be detected in such a metaldeficient system. For galaxies with metallicities between solar and 0.1 solar, Wilson (1995) finds $\alpha \propto Z^{-0.67}$. Extrapolating this relationship to a metallicity of $1 / 41$ solar yields $\alpha \simeq 12 \alpha_{\mathrm{G}}$ and $M\left(\mathrm{H}_{2}\right)<4.9 \times 10^{8} M_{\odot}$ for SBS $0335-052$. However, these estimates are only applicable for dense regions where the $\mathrm{CO}$ molecules could survive the intense ultraviolet radiation field. In regions of low extinction $\left(A_{V} \lesssim 2 \mathrm{mag}\right)$, the global $\mathrm{CO} / \mathrm{H}_{2}$ abundance ratio is expected to be extremely nonlinear with metallicity (Maloney \& Black 1988). 


\section{DISCUSSION}

\subsection{Dust and The Infrared Spectral Energy Distribution}

Thuan et al. (1999) fit the observed mid-infrared spectrum with the profile for a $250 \mathrm{~K}$ extincted blackbody with emissivity $Q_{v} \propto v^{\beta}$, where $\beta=1.5$ is the assumed dust emissivity index. Data at longer wavelengths can help better constrain the infrared spectral energy distribution: in addition to the new flux measurement at $67 \mu \mathrm{m}$, there are upper limits available from $I R A S$ at $12,25,60$, and $100 \mu \mathrm{m}$. These limits are compatible with the levels detected at similar wavelengths (see Fig. 6). With the infrared picture of SBS 0335-052 now more fully developed, it is clear that the data suggest multiple dust components. A fit of the superposition of two blackbodies with emissivity $Q_{v} \propto v^{1.5}$, with variable normalizations and dust temperatures, to the $6.7,7.7,12.5,25$, and $67 \mu \mathrm{m}$ broadband data results in dust temperatures for the two components of $T_{d}=80$ and $210 \mathrm{~K}^{7}$ Varying the emissivity from $Q_{v} \propto v^{1.0}$ to $v^{2.0}$ changes the dust temperatures by a maximum of $\pm 20 \%$. Two relatively warm dust components are also evident in Seyfert galaxies (Pérez García, Rodríguez Espinosa, \& Santolaya Rey 1998) and luminous galaxies such as Arp 220, NGC 6240, and NGC 4038/39 (Klaas et al. 1997). More far-infrared data are needed to verify whether

${ }^{7}$ IRAS marginally detected $(2 \sigma)$ SBS $0335-052$ at $25 \mu \mathrm{m}$. If a $2 \sigma$ $25 \mu \mathrm{m}$ flux is used in the fit instead of a $3 \sigma$ upper limit, the resulting dust temperatures are 70 and $207 \mathrm{~K}$.

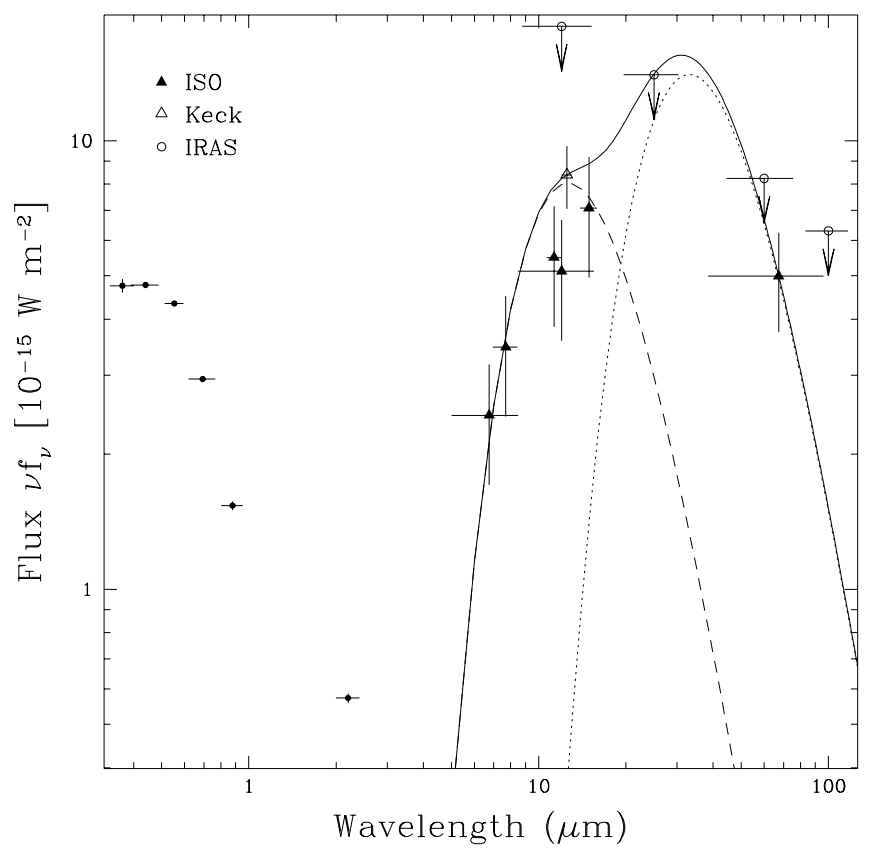

Fig. 6.-Fit to the infrared spectral energy distribution using a superposition of two $Q_{v} \propto v^{1.5}$ blackbodies, one at $T_{d}=210 \mathrm{~K}$ (dashed line) and the other at $T_{d}=80 \mathrm{~K}$ (dotted line). The upper limits are from IRAS and are equal to 3 times the rms deviation of 7-10 scans from the median scan. The five mid-infrared broadband values from Thuan et al. (1999) have been assigned $30 \%$ flux uncertainties since (i) that is the discrepancy between their spectroscopy and broadband photometry, and (ii) 30\% is also the approximate calibration uncertainty for ISO mid-infrared data.
SBS 0335-052 has an additional colder $\left(T_{d} \sim 25 \mathrm{~K}\right)$ dust component.

The density and total amount of dusty material in the core of SBS $0335-052$ determines the central opacity. A simple estimate of the optical depth is the ratio of the observed surface brightness to the intensity of the expected blackbody profile, $\tau \sim f_{v} \Omega^{-1} / B_{v}\left(T_{d}\right)$, where $\Omega$ is the solid angle of the object. The mid-infrared opacity for SBS $0335-052$ is less than $3 \times 10^{-3}$ at $12.5 \mu \mathrm{m}$ for a wide range of hot dust temperatures $\left(T_{d}^{\text {hot }}>110 \mathrm{~K}\right)$. Such an opacity corresponds to $A_{V}<1 \mathrm{mag}$, in agreement with the result of Izotov et al. (1997). Furthermore, the total ionizing luminosity computed from the number of UV photons per second $(\S 4.3)$ is of order $8 \times 10^{8} L_{\odot}$, similar to the midinfrared luminosity (Table 1). These results suggest $\tau_{\mathrm{uv}} \sim 1$, in contrast to the claim for $A_{V} \sim 20 \mathrm{mag}$ by Thuan et al. (1999), and argue that the model of a hot blackbody affected by a large column of extinction is invalid.

Since the infrared radiation is optically thin in emission, dust masses can be directly computed using the infrared fluxes. The infrared flux density may be expressed in terms of the number of grains $N$ of size $a$, density $\rho$, and emissivity $Q_{v}$ radiating at temperature $T_{d}$ at distance $d: f_{v}=$ $N \pi B_{v}\left(T_{d}\right) Q_{v} 4 \pi a^{2} / 4 \pi d^{2}$. And since the dust mass can be expressed as $M_{\mathrm{dust}}=N(4 / 3) \pi a^{3} \rho$, one finds

$$
M_{\mathrm{dust}}=\frac{4 a \rho f_{v} d^{2}}{3 Q_{v} B_{v}\left(T_{d}\right)} \equiv \frac{f_{v} d^{2}}{\kappa_{v} B_{v}\left(T_{d}\right)}
$$

(e.g., Hildebrand et al. 1977). In other words, the dust mass is a function of the observed flux density and the estimated dust temperature and does not depend on the size of the emitting region. If the dust temperature is $210 \mathrm{~K}$ for the hot dust component and the opacity coefficient $\kappa(12 \mu \mathrm{m})=940$ $\mathrm{cm}^{2} \mathrm{~g}^{-1}$ (Li \& Draine 2001), the dust mass computed from the $12.5 \mu \mathrm{m}$ flux is $M_{\mathrm{dust}}^{\text {hot }} \simeq 6 M_{\odot}$. The mass of the cooler dust may be computed from the dust emission at $67 \mu \mathrm{m}$, radiation which is more likely to arise from steady state processes compared to the mid-infrared emission from very small grains and polycyclic aromatic hydrocarbons (PAHs). The total mass of the presumably larger and cooler dust grains in SBS 0335-052, if $T_{d}=80 \mathrm{~K}$ and $\kappa(70 \mu \mathrm{m})=67.3$ $\mathrm{cm}^{2} \mathrm{~g}^{-1}$ ( $\mathrm{Li} \&$ Draine), is $M_{\mathrm{dust}}^{\text {warm }} \simeq 2400 M_{\odot}$. Thus, the warm dust mass is much larger than the hot dust mass in a simple two-component model. We will henceforth use it to represent the total dust mass in SBS 0335-052. Considering that the long-wavelength end of the spectral energy distribution is poorly constrained, the warm dust temperature estimate, and thus the overall dust mass, is highly uncertain. Any cold dust, which is unconstrained by the measurements reported here, would likely dominate the total dust mass.

ISO observations show a mid-infrared spectrum with none of the standard PAH features (Thuan et al. 1999). For a two-component dust model, the hotter dust in SBS $0335-052$ is 400 times less massive than the warm dust. Unless it can be explained by sensitivity arguments, it seems peculiar then that there are no obvious PAH features in its mid-infrared spectrum - typically only regions with very energetic interstellar radiation fields, through destruction and/or ionization processes, lack the ubiquitous midinfrared aromatic emission features (e.g., the core of M 17; Cesarsky et al. 1996). Conversely, emission from merely warm dust should be replete with aromatic features. A possible explanation is that PAHs are thought to condense out 
of the outflows of carbon-rich red giants (Allamandola, Tielens, \& Barker 1989), but the starburst in SBS $0335-052$ is too young $(<7 \mathrm{Myr})$ for stars to have reached this phase - only stars more massive than $\sim 20 M_{\odot}$ are expected to spend less than $10 \mathrm{Myr}$ on the main sequence (e.g., Bressan et al. 1993).

\subsection{The Gas-to-Dust Ratio}

The global gas-to-warm dust mass ratio is relatively straightforward to compute. From the $\mathrm{H}$ I measurement of Pustilnik et al. (2001) of $M(\mathrm{H} \mathrm{I}) \sim 8 \times 10^{8} M_{\odot}$ and the molecular hydrogen mass limit of $M\left(\mathrm{H}_{2}\right)<4.9 \times 10^{8} M_{\odot}$,

$$
\left|\frac{M_{\text {gas }}}{M_{\text {dust }}}\right|_{\text {global }}=\left|\frac{M(\mathrm{H} \mathrm{I})+M\left(\mathrm{H}_{2}\right)}{M_{\text {dust }}}\right|_{\text {global }} \lesssim 10^{5.7} .
$$

This value lies on the high end of the expected range for a compact dwarf galaxy compared with the range $10^{3} \lesssim$ $M_{\text {gas }} / M_{\text {dust }} \lesssim 10^{5}$ typically seen for blue compact dwarfs (Lisenfeld \& Ferrara 1998), and with that usually found in more mundane regions, like the disks of spiral galaxies where $M_{\text {gas }} / M_{\text {dust }} \sim 300$ (see Mayya \& Rengarajan 1997 and references therein).

However, a global gas-to-dust ratio for SBS $0335-052$ is a misleading quantity since the dust and gas are not measured in the same volume. A more sensible constraint on the gas-to-dust mass ratio is derived from the ionized gas mass. The ionized gas density within the Strömgren sphere is $n_{e} \sim 45 \mathrm{~cm}^{-3}(\S 4.3)$. The central $80 \mathrm{pc}$ therefore contains approximately $3 \times 10^{5} M_{\odot}$ of gas, and

$$
\left|\frac{M_{\text {gas }}^{\text {ionized }}}{M_{\text {dust }}}\right|_{r<80 \mathrm{pc}} \gtrsim 100,
$$

representing a lower limit on the central gas-to-dust mass ratio. This estimate is limited by the assumption that the entire $\sim 2400 M_{\odot}$ dust mass falls within the inner 80 pc.

\subsection{Clues from the Bry Emission}

The near-infrared broadband fluxes of starbursts can be dominated by nebular emission lines that unduly influence stellar age estimates (Leitherer \& Heckman 1995). An alternative indicator of the starburst age is the $\mathrm{Br} \gamma$ equivalent width, a parameter that reflects the ratio of young blue massive stars to the evolved red stellar population. For a plausible range of initial mass function parameters, the Starburst99 models (Leitherer et al. 1999) indicate that $E W(\mathrm{Br} \gamma) \simeq 235 \AA$ for $Z_{\odot} / 50$ metallicity material corresponds to a stellar age of 3-6 $\times 10^{6} \mathrm{yr}$. This age holds for both an instantaneous and a continuing star formation model, consistent with the age estimate from other nearinfrared spectroscopic and broadband data (Vanzi et al. 2000).

If the central $\mathrm{H}$ II region is modeled as a Strömgren sphere, it is possible to use the size of the $\mathrm{Br} \gamma$ emission and the $\mathrm{Br} \gamma$ luminosity to infer the density of the ionized gas $n_{e}$ (see Table 2). For an intrinsic $\mathrm{Br} \alpha$-to-Br $\gamma$ luminosity ratio of 2.9 , the $\mathrm{Br} \gamma$ luminosity for SBS $0335-052$ corresponds to

$$
\begin{aligned}
N_{\mathrm{uv}} & =\frac{N_{\mathrm{uv}}}{N_{\mathrm{Br} \alpha}} \frac{N_{\mathrm{Br} \alpha}}{N_{\mathrm{Br} \gamma}} N_{\mathrm{Br} \gamma}=\frac{N_{\mathrm{uv}}}{N_{\mathrm{Br} \alpha}} \frac{L_{\mathrm{Br} \alpha} \lambda_{\mathrm{Br} \alpha}}{L_{\mathrm{Br} \gamma} \lambda_{\mathrm{Br} \gamma}} \frac{L_{\mathrm{Br} \gamma}}{h v_{\mathrm{Br} \gamma}} \\
& \sim 1.5 \times 10^{53},
\end{aligned}
$$

TABLE 2

SIZES OF SBS $0335-052$

\begin{tabular}{lcc}
\hline \hline Wavelength & $\begin{array}{c}\text { Size } \\
(\operatorname{arcsec})\end{array}$ & $\begin{array}{c}\text { Size } \\
(\mathrm{kpc})\end{array}$ \\
\hline $2.166 \mu \mathrm{m}(\mathrm{Br} \gamma)$ & $\sim 1 \times 1$ & $0.26 \times 0.26$ \\
$2.2 \mu \mathrm{m}$ & $\sim 2 \times 1$ & $0.52 \times 0.26$ \\
$12.5 \mu \mathrm{m}$ & $0.31 \times 0.31$ & $0.08 \times 0.08$ \\
$20 \mathrm{~cm}^{\mathrm{a}}$ & $<6 \times 6$ & $1.55 \times 1.55$ \\
\hline
\end{tabular}

${ }^{\text {a }}$ Unresolved.

ionizing photons per second (see Ho, Beck, \& Turner 1990), or approximately 2800 O5 stars (e.g., Panagia 1973). Since the volume of the Strömgren sphere can be expressed as $(4 / 3) \pi R_{\mathrm{Br} \gamma}^{3}=N_{\mathrm{uv}} / \alpha_{\mathrm{B}} n_{e}^{2}$, for a measured $R_{\mathrm{Br} \gamma}=130 \mathrm{pc}$ the inferred density is $n_{e} \sim 45 \mathrm{~cm}^{-3}$, reasonable for $\mathrm{H}$ II regions (Osterbrock 1989). Here we have assumed Case B recombination (with $\alpha_{\mathrm{B}} \simeq 2.6 \times 10^{-13} \mathrm{~cm}^{-3} \mathrm{~s}^{-1}$ ) for a nebula at temperature $T_{e}=10,000 \mathrm{~K}$, but the same electron density is found to within a factor of 2 for a wide temperature range.

Inasmuch as $\mathrm{Br} \gamma$ emission is associated with $\mathrm{H}$ II regions, it is indicative of the heating input to the interstellar medium; the $\mathrm{Br} \gamma$ flux can be related to the thermal radio flux density $f_{v}^{\text {th }}$. Following Ho et al. (1989) and assuming a radio spectral slope of -0.1 appropriate for optically thin free-free emission from ionized gas, we estimate the expected thermal radio continuum flux at $20 \mathrm{~cm}$ from

$$
f_{v}^{\mathrm{th}}(20 \mathrm{~cm}) \approx 1.0 \mathrm{mJy}\left(\frac{d}{\mathrm{Mpc}}\right)^{-2}\left(\frac{N_{\mathrm{uv}}}{10^{50} \mathrm{~s}^{-1}}\right) .
$$

The number of ionizing photons per second inferred from the integrated $\mathrm{Br} \gamma$ flux for SBS $0335-052$ predicts $f_{v}^{\text {th }}(20$ $\mathrm{cm}) \approx 0.5 \mathrm{mJy}$, approximately the observed value for $f_{v}(20$ $\mathrm{cm}$ ). Since the prediction is at least as large as the observed $20 \mathrm{~cm}$ flux density, the straightforward interpretation is that there is little or no evidence for nonthermal radio contributions - there has been, at most, a low level of supernova activity in the short star formation history of SBS $0335-052$. A quick $5 \mathrm{GHz}$-array observation with the VLA could easily verify whether the radio source has a flat spectrum indicative of $\mathrm{H}$ II regions or a steep spectrum produced by supernova remnants. For comparison, the global thermal fraction of the flux density at $20 \mathrm{~cm}$ in normal star-forming galaxies is about 10\% (Condon 1992). According to Matteucci \& Recchi (2001), the peak in the supernovae rate (and thus the maximum metal enrichment rate) for an instantaneous starburst episode does not occur until 40-50 Myr after the onset of star formation. Such an upper limit to the age of the starburst in SBS 0335-052 agrees with the constraints placed by the $\mathrm{Br} \gamma$ data. Furthermore, a scenario of modest prior supernova activity would also be consistent with the interpretation laid out in $\S 4.4$ for the high infrared-to-radio ratio for SBS $0335-052$.

\subsection{The Infrared-to-Radio Ratio}

The radio continuum flux for SBS $0335-052$ spatially coincides with its emission at $12.5 \mu \mathrm{m}$. Thus, if the emission in the mid- and far-infrared is spatially coincident, the farinfrared-to-radio ratio is $f_{v}(67 \mu \mathrm{m}) / f_{v}(1.49 \mathrm{GHz}) \simeq 280$. This flux density ratio is compatible with the typical ratio for normal star-forming galaxies, which is approximately 
$200_{-70}^{+120}$. Note, however, that in the absence of IRAS detections for SBS $0335-052$ an ISO-based $f_{v}(67 \mu \mathrm{m})$ has been used as a proxy for the typical IRAS-based definition for the far-infrared "FIR" flux (Helou et al. 1988), which effectively recovers the entire $42-122 \mu \mathrm{m}$ far-infrared flux; the ISOPHOT filter used here, on the other hand, has a shorter central wavelength and an overall narrower profile $(\S 2.5)$ and thus does not provide as robust an estimate of the bolometric infrared flux. The FIR 42-122 $\mu \mathrm{m}$ flux for SBS 0335-052 computed from the model spectral energy distribution shown in Figure 6 is $5.4 \pm 1.1 \times 10^{-15} \mathrm{~W}$ $\mathrm{m}^{-2}$, and thus the logarithmic measure defined by Helou, Soifer, \& Rowan-Robinson (1985) is

$$
\begin{aligned}
q & =\log \left[\frac{\mathrm{FIR}}{3.75 \times 10^{12} \mathrm{~W} \mathrm{~m}^{-2}}\right]-\log \left[\frac{f_{v}(1.49 \mathrm{GHz})}{\mathrm{W} \mathrm{m}^{-2} \mathrm{~Hz}^{-1}}\right] \\
& \simeq 2.56 \pm 0.09 .
\end{aligned}
$$

The $20 \%$ uncertainty is conservatively derived from the full range of FIR model fluxes found after invoking different combinations of the $25 \mu \mathrm{m}$ flux upper limit ( 2 to $3 \sigma$ ) and the dust emissivity index $(1.0 \lesssim \beta \lesssim 2.0)$. In other words, the far-infrared-to-radio ratio estimated from the model spectral energy distribution is $\simeq 360 \pm 70$, slightly outside the 1 $\sigma$ range for normal star-forming galaxies.

Given its youth, small size, and the inferred lack of nonthermal radio flux, it is surprising that SBS 0335-052 exhibits a fairly "normal" infrared-to-radio ratio. In resolved sources, the radio continuum emission is usually more diffuse than the infrared light (Marsh \& Helou 1998) and is explained by the spreading of cosmic rays as they decay and are trapped in magnetic field lines (Helou \& Bicay 1993). The smearing scale length in normal galaxies is many hundreds of parsecs (Marsh \& Helou 1998), much larger than the 80 pc mid-infrared size of SBS 0335-052. Moreover, the relatively small disk scale heights for dwarf galaxies may result in cosmic rays escaping comparatively easily (Helou \& Bicay 1993). Finally, the youth of SBS 0335-052 means that relatively few supernovae have occurred, resulting in low cosmic ray production and weak interstellar magnetic fields. All these lines of reasoning predict a low nonthermal radio continuum flux for SBS 0335-052 compared to the far-infrared flux, consistent with the findings using the $\mathrm{Br} \gamma$ data. In short, since the canonical far-infrared-to-radio ratio presumably hinges on a typically strong $(\sim 90 \%)$ nonthermal contribution to the radio flux, the apparently negligible nonthermal radio emission of SBS $0335-052$ should result in an extremely high far-infrared-to-radio ratio.

Given the elevated mid- to far-infrared ratio and the unusual infrared spectral energy distribution of SBS 0335-052, perhaps it is more appropriate to instead compute a total infrared-to-radio ratio. In normal galaxies the far-infrared flux is a useful measure of the total infrared flux (TIR), with FIR typically half of the $3-1100 \mu \mathrm{m}$ bolometric infrared flux (Dale et al. 2001): TIR/radio $\sim 400_{-150}^{+230}$. But the conspicuously intense interstellar radiation field of SBS $0335-052$ results in an unusually strong contribution at mid-infrared wavelengths - the FIR flux for SBS 0335-052 computed from the model spectral energy distribution shown in Figure 6 (and all plausible variations) is less than one-fourth the model's bolometric infrared flux, resulting in TIR/radio $\sim 1600 \pm 300$. In short, the total infrared to radio flux for SBS $0335-052$ is about 4 times that in normal star-forming galaxies and is likely due to a relative lack of trapped interstellar cosmic rays, resulting in comparatively weak radio emission.

\subsection{The Intensity of the Interstellar Radiation Field}

In a detailed study of the $\mathrm{N} 4$ complex of $\mathrm{H}$ iI regions in the Large Magellanic Cloud, Contursi et al. (1998) show how the mid-infrared color $f_{v}(15 \mu \mathrm{m}) / f_{v}(7 \mu \mathrm{m})$ strongly varies as a function of interstellar radiation field intensity, with the highest ratios $\left[f_{v}(15 \mu \mathrm{m}) / f_{v}(7 \mu \mathrm{m}) \gtrsim 4\right]$ corresponding to ultraviolet radiation densities approaching $10^{4}$ times the interstellar radiation field in the solar neighborhood. Though the distance to SBS 0335-052 precludes such a spatially detailed analysis, the average interstellar heating intensity can be estimated from $\langle U\rangle \sim L(12.5 \mu \mathrm{m}) /$ $4 \pi r^{2} \approx 15$ ergs $\mathrm{cm}^{-2} \mathrm{~s}^{-1} \simeq 10,000 G_{o}$, where $G_{o}=1.6$ $\times 10^{-3} \mathrm{ergs} \mathrm{cm}^{-2} \mathrm{~s}^{-1}$ and $1.7 G_{o}$ is the flux of the local interstellar far-ultraviolet radiation field (Hollenbach \& Tielens 1999 and references therein). This result is consistent with the findings of Contursi et al. $(1998,2000)$ : an unusually high mid-infrared color, $f_{v}(15 \mu \mathrm{m}) / f_{v}(7 \mu \mathrm{m}) \approx 6.4$ in the case of SBS 0335-052 (Thuan et al. 1999), corresponds to an interstellar radiation field at least 4 orders of magnitude greater than the local value. Moreover, a reasonable dust temperature is found for SBS 0335-052 if it is assumed that $\langle U\rangle \propto T_{d}^{4+\beta}$. Scaling from the $\sim 17.5 \mathrm{~K}$ dust temperature found for diffuse Galactic cirrus (Boulanger et al. 1996), an order-of-magnitude estimate for the interstellar dust temperature for such a radiation field is $T_{d} \sim(17.5$ $\mathrm{K})\left(10^{4} G_{o} / G_{o}\right)^{1 /(4+\beta)} \sim 100 \mathrm{~K}$. Though this result is compatible with the dust temperature predicted for the bulk of the interstellar medium in SBS 0335-052 (§ 4.1), it is the hot dust that is primarily emitting at $12.5 \mu \mathrm{m}$. One interpretation of this discrepancy is that the small grain emission at mid-infrared wavelengths results from a semistochastic process; the small grain emission is perhaps not well represented by a hot blackbody (e.g., Draine \& Anderson 1985).

\subsection{The Compact Nature of the Starburst Region}

The mid-infrared data presented here, with angular resolution better than 0.4 , represent the highest spatial resolution achieved to date in probing the blue compact dwarf galaxy SBS $0335-052$. SBS $0335-052$ is compact at $12.5 \mu \mathrm{m}$ compared with its stellar and gaseous constituents. In normal star-forming galaxies the extent of the midinfrared, far-infrared, and submillimeter emission is comparable to the optical size (Dale et al. 2000; Rice et al. 1988; Chini et al. 1995), and detailed modeling of the optical and near-infrared emission from normal late-type spiral galaxies shows that dust scale lengths typically are larger than that of the stars and that dust radially extends to larger distances than stars in the disk (Xilouris et al. 1999). On the other hand, the high angular resolution studies of Soifer et al. (2000) and Soifer et al. (2001) show that it is common for both ultraluminous and starburst galaxies to be particularly compact at $12 \mu \mathrm{m}$ compared with their optical distributions, especially for ULIRGs, with typically more than $50 \%$ of the mid-infrared emission arising from $<100-300 \mathrm{pc}$ sized regions. Thus, perhaps it is relatively common for actively star-forming systems to produce the bulk of their thermal mid-infrared emission in small volumes surrounding a handful of bright super star clusters. This may be 
especially true for dwarf systems like SBS 0335-052, where a relatively small stellar population may be dominated by a few extremely bright stars or star clusters.

The lack of extended hot dust emission in SBS 0335-052 is most easily attributed to geometry: the relatively small number of bright stars in SBS $0335-052$ heat to high temperatures only the most proximate dust. As outlined in $\S 4.1$, the mass of this hot dust is $\sim 6 M_{\odot}$. The bulk of the $\sim 2400$ $M_{\odot}$ of dust spread throughout the rest of the interstellar medium is located significantly farther from the hottest stars and is thus only warmed to $\sim 80 \mathrm{~K}$ on average. Balancing the radiation absorbed and reemitted by dust grains yields the following simple relation for the approximate temperature of dust grains in thermal equilibrium with the surrounding interstellar radiation field:

$$
T_{d}=109 \mathrm{~K}\left(\frac{Q_{\mathrm{uv}}}{Q_{\mathrm{ir}}}\right)^{1 / 4}\left(\frac{d}{1 \mathrm{pc}}\right)^{1 / 2}\left(\frac{L_{\mathrm{uv}}}{10^{9} L_{\odot}}\right)^{1 / 4},
$$

where $Q_{\mathrm{uv}}$ and $Q_{\mathrm{ir}}$ are the absorption and emission coefficients in the ultraviolet and infrared, respectively. If the dust emission is optically thin and distributed in a circularly symmetric fashion centered on the $10^{9} L_{\odot}$ ionizing luminosity source (e.g., the brightest super star cluster), then the hot dust is $\sim 3 \mathrm{pc}$ from the ionizing source and the warm dust lies at a characteristic distance of $\sim 15 \mathrm{pc}$ (assuming $Q_{\lambda} \propto \lambda^{-1.5}$ ). A substantial portion of the ionizing photons from the star clusters, on the other hand, encounter neutral gas at much larger distances, and that is why the central $\mathrm{H}$ II region in SBS 0335-052 spans more than $1 \mathrm{kpc}$ (Izotov et al. 1997). Needless to say, the angular resolution of the extant infrared data is at least an order of magnitude too low to corroborate this scenario.

In many respects, SBS 0335-052 resembles NGC 5253: it is a low-metallicity dwarf galaxy dominated by a $<10 \mathrm{Myr}$ old compact and strong mid-infrared emitting star-forming region for which the radio continuum emission is almost entirely thermal (Beck et al. 1996; Gorjian, Turner, \& Beck 2001). Can a useful comparison be made between the star-forming regions in NGC 5253 and SBS 0335-052? Probably not, in that the mid-infrared size of SBS $0335-052$ is truly extended and is not confined to an ultracompact 1-2 pc super star cluster containing several thousand O stars as in NGC 5253 (Turner, Beck, \& Ho 2000) - the stellar component of SBS 0335-052 is comprised of multiple star clusters spread throughout the inner $520 \mathrm{pc}$ (Thuan et al. 1997). Moreover, even though its midinfrared emission appears to originate from a quite compact region, the corresponding volume and stellar density in the core of SBS $0335-052$ are much less extreme than for the super star cluster in NGC 5253. Finally, the potential for future star formation is substantially more promising for SBS 0335-052: the galaxy has a large $\mathrm{H}$ i reservoir that spans $66 \mathrm{kpc}$ by $22 \mathrm{kpc}$ (covering both the eastern and western optical components of SBS 0335-052), an order of magnitude larger in linear extent than the optical emission.

\section{SUMMARY}

The previous work on SBS 0335-052 and our new results suggest that SBS $0335-052$ is a galaxy observed early in its formative years and is likely experiencing its first burst of star formation. We find that:

1. SBS $0335-052$ has a very compact distribution of hot dust, which suggests that significant star formation has not occurred for long nor over large spatial scales. A more extended dust distribution presumably requires more red giant stars processing dust grains for a longer time.

2 . The rough spectral shape mapped out by the available mid- and far-infrared fluxes suggests that the global distribution of dust is in the form of at least one warm $(80 \mathrm{~K})$ component with a mass of $\sim 2400 M_{\odot}$ and one $\sim 6 M_{\odot}$ hot $(210 \mathrm{~K})$ component; more longer wavelength data are needed to verify the presence of a substantial cold dust component.

3. In spite of the depressed global metallicity, hot dust is one of the energetically dominant features of the galaxy's interstellar medium, resulting in the mid-infrared emission being remarkably comparable to that in the far-infrared. An important conclusion may be reached here: young systems that are characterized by a few pockets of active star formation and an overall metal-poor interstellar medium will show elevated mid- to far-infrared ratios. Perhaps normal characteristics are achieved only after a certain amount of time has passed, after large dust grains have had time to form or build up. Only then will the more extended, cooler far-infrared dust emission come to dominate the pockets of hot dust emission.

4. The mid-infrared flux coupled with the mid-infrared size and dust temperature indicate that the dust in the starforming region is optically thin in emission and $A_{V}<1$ mag. Since CO molecules likely need to be self-shielding and thus optically thick to be found in appreciable amounts (Meier et al. 2001), it is not surprising that $\mathrm{CO}$ emission was not detected for SBS $0335-052$.

5. The galaxy has a bolometric infrared-to-radio ratio that is 4 times what is expected for normal star-forming galaxies. This is presumably due to a lack of cosmic rays trapped by interstellar magnetic field lines, as evidenced by the galaxy's negligible nonthermal radio emission. The dearth of trapped cosmic rays is explained by a combination of two effects: (i) the relatively short star formation history of this galaxy - the starburst is so young that its luminosity is still dominated by extremely massive, luminous stars. The infrared luminosity output per star is high, but the number of supernova remnants is low, assuming the radio power per supernova remnant is independent of progenitor mass; (ii) the compact size of SBS 0335-052dwarf galaxies are known to exhibit low trapping of cosmic rays (Condon, Anderson, \& Helou 1991).

6. SBS $0335-052$ has an extremely large Bry equivalent width that is indicative of a vigorous star-forming region only 3-6 Myr old. The distribution of the $\mathrm{Br} \gamma$ hydrogen recombination line appears to be more extended than the mid-infrared continuum.

7. The estimated average interstellar heating intensity is $\sim 10,000$ times the intensity found in the solar neighborhood. Comparable heating intensities are not typically seen globally in galaxies; only $\mathrm{H}$ II regions show such elevated heating intensities.

We are happy to acknowledge M. Sauvage for the generous donation of unpublished $I S O$ results. We are also grateful T. Murphy helped with the LWS observations and the PIFS data reduction, and for the assistance E. Egami provided with the PIFS observing run. The comments from an anonymous referee helped to improve the text. The W. M. 
Keck Observatory is operated as a scientific partnership between the California Institute of Technology, the University of California and the National Aeronautics and Space Administration. It was made possible by the generous financial support of the W. M. Keck Foundation. ISO is an ESA project with instruments funded by ESA member states (especially the PI countries: France, Germany, The
Netherlands and the United Kingdom) and with the participation of ISAS and NASA. This research has made use of the NASA/IPAC Extragalactic Database, which is operated by JPL/Caltech, under contract with NASA. B. T. S. is supported by grants from the NSF and NASA. D. T. F. acknowledges support from NSF grant AST 96-13717 made to the Owens Valley Millimeter Array.
Allamandola, L. J., Tielens, A. G. G. M., \& Barker, J. R. 1989, ApJS, 71, 733

Beck, S. C., Turner, J. L., Ho, P. T. P., Lacy, J. H., \& Kelly, D. M. 1996 , ApJ, 457, 610

Beichman, C. A., Neugebauer, G., Habing, H. J., Clegg, P. E., \& Chester, T. J. 1985, IRAS Catalog and Atlases, Explanatory Supplement (Washington: GPO)

Bessel, M. S. 1979, PASP, 91, 589

Boulanger, F., Abergel, A., Bernard, J.-P., Burton, W. B., Desert, F.-X., Hartmann, D., Lagache, G., \& Puget, J.-L. 1996, A\&A, 312, 256

Bressan, A., Fagotto, F., Bertelli, G., \& Chiosi, C. 1993, A\&AS, 100, 647

Cesarsky, D., Lequeux, J., Abergel, A., Pérault, M., Palazzi, E., Madden, S., \& Tran, D. 1996, A\&A, 315, L309

Chini, R., Kruegel, E., Lemke, R., \& Ward-Thompson, D. 1995, A\&A, 295, 317

Cole, S., Lacey, C. G.,Baugh, C. M., \& Frenk, C. S. 2000, MNRAS, 319, 168 Condon, J. J. 1992, ARA\&A, 30, 575

Condon, J. J., Anderson, M. L., \& Helou, G. 1991, ApJ, 376, 95

Condon, J. J., Cotton, W. D., Greisen, E. W., Yin, Q. F., Perley, R. A., Taylor, G. B., \& Broderick, J. J. 1998, AJ, 115, 1693

Contursi, A., et al. 1998, A\&A, 336, 662

Contursi, A., et al. 2000, A\&A, 362, 310

Dale, D. A., et al. 2000, AJ, 120, 583

Dale, D. A., Helou, G., Contursi, A., Silbermann, N. A., \& Kolhatkar, S. 2001, ApJ, 549, 215

Draine, B. T., \& Anderson, N. 1985, ApJ, 292, 494

Gorjian, V., Turner, J. L., \& Beck, S. C. 2001, preprint (astro-ph/0103101)

Helou, G., \& Bicay, M. D. 1993, ApJ, 415, 93

Helou, G., Khan, I. R., Malek, L., \& Boehmer, L. 1988, ApJS, 68, 151

Helou, G., Soifer, B. T., \& Rowan-Robinson, M. 1985, ApJ, 298, L7

Helou, G., et al. 1996, A\&A, 315, L157

Hildebrand, R. H., Whitcomb, S. E., Winston, R., Stiening, R. F., Harper,

D. A., \& Moseley, S. H. 1977, ApJ, 216, 698

Ho, P. T. P., Beck, S. C., \& Turner, J. L. 1990, ApJ, 349, 57

Ho, P. T. P., Turner, J. L., Fazio, G. G., \& Willner, S. P. 1989, ApJ, 344, 135

Hollenbach, D. J., \& Tielens, A. G. G. M. 1999, Rev. Mod. Phys., 71, 173

Izotov, Y. I., Lipovetsky, V. A., Chaffee, F. H., Foltz, C. B., Guseva, N. G., \& Kniazev, A. Y. 1997, ApJ, 476, 698

Jones, B., \& Puetter, R. C. 1993, Proc. SPIE, 1946, 610

Klaas, U., Haas, M., Heinrichsen, I., \& Schulz, B. 1997, A\&A, 325, L21

Langston, G. I., Conner, S. R., Heflin, M. B., Lehar, J., \& Burke, B. F. 1990, ApJ, 353, 34
Leitherer, C., \& Heckman, T. M. 1995, ApJS, 96, 9

Leitherer, C., et al. 1999, ApJS, 123, 3

Li, A., \& Draine, B. T. 2001, ApJ, 554, 778

Lisenfeld, U., \& Ferrara, A. 1998, ApJ, 496, 145

Lucy, L. B. 1974, AJ, 79, 745

Maloney, P., \& Black, J. H. 1988, ApJ, 325, 389

Marsh, K. A., \& Helou, G. 1998, ApJ, 493, 121

Matteucci, F., \& Recchi, S. 2001, ApJ, in press (astro-ph/0105074)

Matthews, K., \& Soifer, B. T. 1994, Exp. Astron., 3, 77

Mayya, Y. D., \& Rengarajan, T. N. 1997, AJ, 114, 946

Meier, D. S., Turner, J. L., Crosthwaite, L. P., \& Beck, S. 2001, AJ, 121, 740

Murphy, T. W., Jr., Matthews, K., \& Soifer, B. T. 1999, PASP, 111, 1176

Murphy, T. W., Jr., Soifer, B. T., Matthews, K., \& Armus, L. 2000, AJ, 120 , 1675

Oliva, E., \& Origlia, L. 1992, A\&A, 254, 466

Osterbrock, D. 1989, Astrophysics of Gaseous Nebulae and Active Galactic Nuclei (Herndon: University Science Books)

Panagia, N. 1973, AJ, 78, 929

Papaderos, P., Izotov, Y. I., Fricke, K. J., Thuan, T. X., \& Guseva, N. G. 1998, A\&A, 338, 43

Pérez García, A. M., Rodríguez Espinosa, J. M., \& Santolaya Rey, A. E. 1998, ApJ, 500, 685

Persson, S. E., Murphy, D. L., Krzeminski, W., Roth, M., \& Reike, M. J. 1998, AJ, 116, 2475

Pustilnik, S. A., Brinks, E., Thuan, T. X., Lipovetsky, V. A., \& Izotov, Y. I. 2001, AJ, 121, 1413

Rice, W., Lonsdale, C. J., Soifer, B. T., Neugebauer, G., Kopan, E. L., Lloyd, L. A., de Jong, T., \& Habing, H. J. 1988, ApJS, 68, 91

Sage, L. J., Salzer, J. J., Loose, H.-H., \& Henkel, C. 1992, A\&A, 265, 19

Schlegel, D. J., Finkbeiner, P. F., \& Davis, M. 1998, ApJ, 500, 525

Soifer, B. T., et al. 2000, AJ, 119, 509

Soifer, B. T., et al. 2001, submitted

Thuan, T. X., Izotov, Y. I., \& Lipovetsky, V. A. 1997, ApJ, 477, 661

Thuan, T. X., \& Martin, G. E. 1981, ApJ, 247, 823

Thuan, T. X., Sauvage, M., \& Madden, S. 1999, ApJ, 516, 783

Turner, J. L., Beck, S. C., \& Ho, P. T. P. 2000, ApJ, 532, L109

van Dokkum, P. G., Franx, M., Fabricant, D., Kelson, D. D., \& Illingworth, G. 1999, ApJ, 520, L95

Vanzi, L., Hunt, L. K., Thuan, T. X., \& Izotov, Y. I. 2000, A\&A, 363, 493

Wilson, C. D. 1995, ApJ, 448, L97

Xilouris, E. M., Byun, Y. I., Kylafis, N. D., Paleologou, E. V., \& Papamastorakis, J. 1999, A\&A, 344, 868 